\title{
The Looped Light of the Triple-Slit Real Experiment as a Confirmation for the Extra Dimensions of Quantum Spacetime and the Reality of Dark Energy
}

\author{
Mohamed S. El Naschie \\ Department of Physics, University of Alexandria, Alexandria, Egypt \\ Email: Chaossf@aol.com
}

How to cite this paper: El Naschie, M.S. (2017) The Looped Light of the Triple-Slit Real Experiment as a Confirmation for the Extra Dimensions of Quantum Spacetime and the Reality of Dark Energy. Optics and Photonics Journal, 7, 19-26. https://doi.org/10.4236/opj.2017.72003

Received: February 3, 2017

Accepted: February 11, 2017

Published: February 14, 2017

Copyright $\odot 2017$ by author and Scientific Research Publishing Inc. This work is licensed under the Creative Commons Attribution International License (CC BY 4.0). http://creativecommons.org/licenses/by/4.0/

\section{Open Access}

\begin{abstract}
Looped light in a recent sophisticated real three-slit experiment is interpreted as a strong justification of the old theoretical Peano-Hilbert spacetime model of Ord and El Naschie. This in turn entails the existence of extra fractal dimensions and consequently of real dark energy density in full agreement with previous analysis as well as accurate measurements and observations of COBE, WMAP, and the type 1a supernova.
\end{abstract}

\section{Keywords}

Looped Light, Dark Energy, E-Infinity, Cantorian Spacetime, Peano-Hilbert Spacetime Model, Triple-Slit Experiment, Fractal Kaluza-Klein Theory, WMAP, Type La Supernova

\section{Introduction}

A remarkable experiment conducted recently [1] [2] [3] reveals apparently for the first time clear cut evidence for a looped travelling path of light [1]. In the present work we argue that this is not a rare event but is what the quantum fine structure of spacetime itself imposes on the movement of light's photons. Consequently the said experiment is interpreted as confirmation of the Cantorian fractal proposal [4] [5], which is based upon the Peano-Hilbert spacetime model presented in the eighties of the last century [6] [7] [8]. There is much evidence, both direct and indirect proving the reality of fractal spacetime [8] [9] [10] [11] [12]. However so far none is as convincing as the results presented recently in [1] [2] [3]. Based on the above we can state that once the fractal Peano-Hilbert model of spacetime is accepted as a fact within the framework of the present pa- 
per then the physical reality of extra spacetime dimensions and consequently dark energy and its 95.5 percent density follows suit as a natural by product of the theory [9] [10].

\section{The Peano-Hilbert Model of Spacetime and the Findings of the Recent Triple-Slit Experiment}

The objective of the present paper is to link the result of a recent experiment to the fractal geometry of micro-spacetime [1]-[10] as well as the issue of cosmic dark energy and the accelerated expansion of the universe [9] [14]. In several early publications [6] [7] [8] going back to the eighties and nineties of the last century the author utilized a model due to the Canadian-English physicist G. Ord [7] to argue for the Cantorian fractal nature of quantum spacetime [4] [8] [9] [10]. An early example of this effort bares a striking resemblance to the recent experimental finding of Ref. [1]. Rather than repeating the mathematical analysis of that paper we think nothing can be simpler and more convenient for the reader than comparing Figure 1 of Ref. [3] with Figure 2 of our earlier publication, Ref. [8] (see Figure 1 and Figure 2 of the present paper). However we must stress that the looping of light is not a rare event but rather the very structure of spacetime at the quantum scale. In other words the experiment in Ref. [1] merely used various refined and ingenious techniques by which the micro structure was blown up to the more pronounced picture shown in the results of Ref. [1]. In this way we could state with a high degree of confidence that the triple-slit experiment of Ref. [1] has established the fractal structure of spacetime as a physical reality and not only a theoretical possibility [9]-[21].

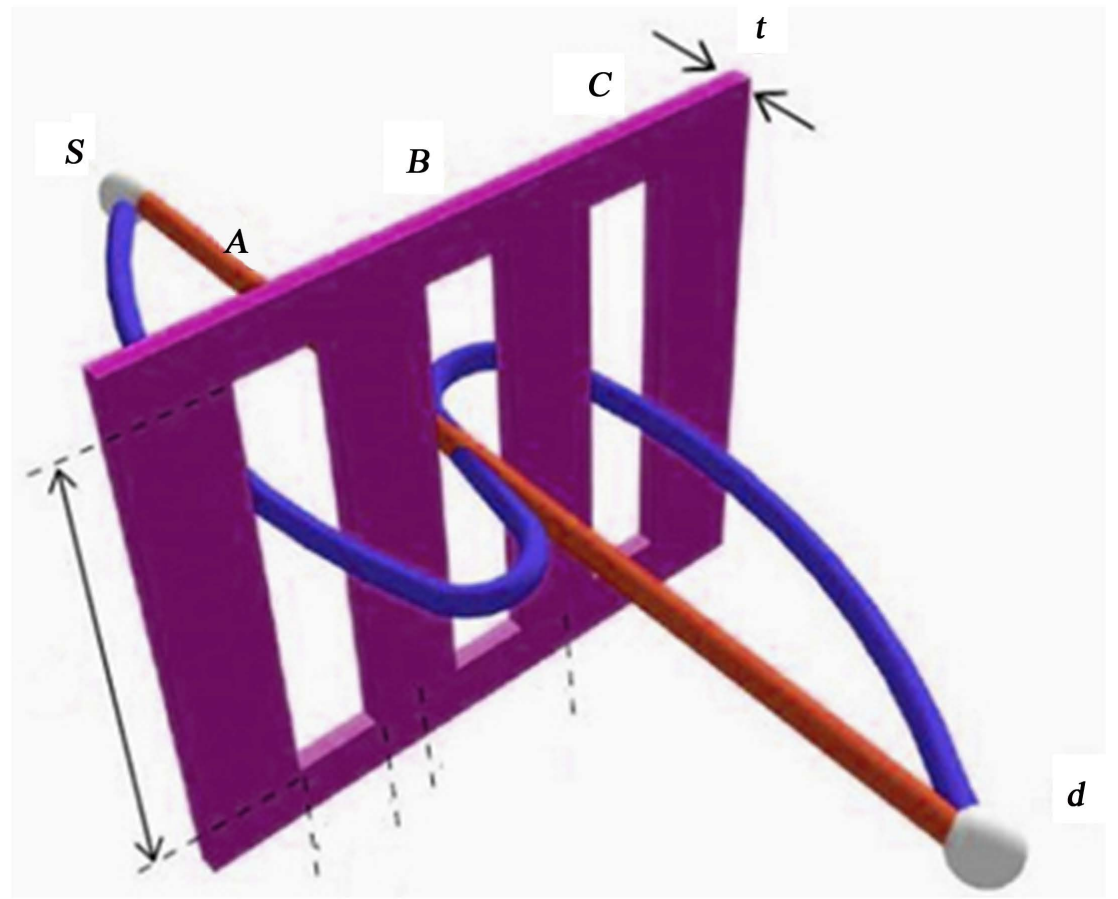

Figure 1. Looped light path in the triple-slit real experiment following Figure 1 of Ref. [3]. 


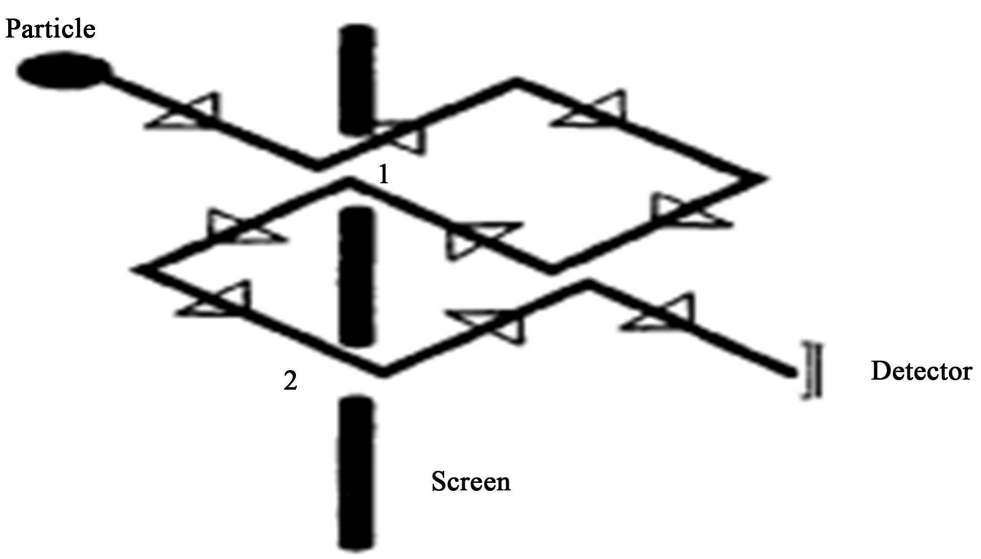

Figure 2. The theoretical light path in the two slit gedanken, (i.e. thought) experiment in a Peano-Hilbert spacetime following Figure 2 of Ref. [8].

The author remembers vividly how Nobel Laureate Ilya Prigogine [11] used to say to him that he liked his model but did not know if spacetime is fractal or not. For this reason many experiments were planned and discussed theoretically but were not carried out [12]. Thus the findings of Ref. [1] at long last achieved what we were trying for years to achieve, namely to experimentally establish the fractal structure of quantum spacetime [12] [13] [14] [15] [16].

\section{The Topological Speed of Light and the Topological Mass of the Universe}

There are many ways to argue that the Kaluza-Klein spacetime dimensionality $D=5$ when extended to a fractal one $D=5+\phi^{3}$ where $\phi=(\sqrt{5}-1) / 2$ represents a total topological mass [13] [14] [15] [16]. It is also easily reasoned that while the integer part $D_{1}=5$ represents pure spacetime quantum wave like topological mass, the irrational part $D_{2}=\phi^{3}$ represents the quantum particlelike topological mass [13] [15]. Seen that way it becomes a truly simple task to find that the energy density is made up of two parts. The first is that proportional to $D_{1}=5$ and is given by [15]

$$
\begin{aligned}
\gamma_{1} & =(5)\left(\frac{1}{2}\right)(V \rightarrow c=\phi)^{2} \\
& =5 \phi^{2} / 2
\end{aligned}
$$

where $c=\phi$ is the topological speed of light [9] [10] [13] [14] [15] [16]. In other words $\gamma_{1}$, is nothing but the dark energy density of pure topological spacetime [9] [10]

$$
\gamma_{1}=\gamma(D)
$$

For the second part $D_{2}=\phi^{3}$ on the other hand one finds in an analogous way that [9] [10] [13] [14] [15] [16]

$$
\begin{aligned}
\gamma_{1} & =\left(\gamma^{3}\right)\left(\frac{1}{2}\right)(V \rightarrow c=\phi)^{2} \\
& =\phi^{5} / 2 .
\end{aligned}
$$


Thus $\gamma_{2}$ is nothing but the ordinary energy density of the quantum particle [9] [10]

$$
\gamma_{2}=\gamma(O)
$$

Adding $\gamma_{1}$ and $\gamma_{2}$ one finds the maximal density $\gamma_{(\max )}=1$ which is expected on the basis of Einstein's well known maximal energy [13] [14] [15] [16]

$$
E=m c^{2} \text {. }
$$

\section{Derivation of $D=5+\phi^{3}$}

The fact that $D=4$ is not sufficient because every point in $D=4$ should also be $D=4$ although being a point means $D=0$ is a subject which we discussed at length and resolved by setting [9] [10] [15]

$$
\begin{aligned}
D & =4+\phi^{3} \\
& 4+\frac{1}{4+\frac{1}{4 \cdots}} \\
& =4+\phi^{3} .
\end{aligned}
$$

On the other hand unification of Einstein's gravity with electromagnetism requires that $D=5 \quad$ [15]. Consequently we can state that [9] [10]

$$
D=4 \rightarrow D=4+1=5
$$

implies that

$$
D=4+\phi^{3} \rightarrow D=4+\phi^{3}+1=5+\phi^{3} .
$$

Here the extra dimension could be regarded as embedding $4+\phi^{3}$ or add one for the volume, similar to what we do in high energy physics when counting particles and matching them to the number of isometries corresponding to say a Lie symmetry group manifold [9] [10].

\section{The Main Results in a Nutshell}

Even a fleeting look at the results of the recent triple-slit experiment looped path of the light [1] [2] [3] reveals its resemblance to the Peano-Hilbert spacetime model proposed decades ago implicitly by G. Ord [7] and explicitly by the present author [4] [5] [6]. A deeper subsequent look reveals even much more and one realizes that the looped path is far from being exotic. In fact it is generic and all that the excellent experimental team managed to do is amplify the fractal structure of spacetime to the extent of almost direct observation [1] [2] [3].

Having established this crucial point we go on to discriminate between two parts that make up our $D=5+\phi^{3}$ spacetime fractal version of Kaluza-Klein. This is [15]

$$
\begin{aligned}
D & =D+D_{2} \\
& =5+\phi^{3} .
\end{aligned}
$$

Here $D_{1}=5$ is related to dark energy via the Einstein equation $E=m c^{2}$ and is given by [13] [14] [15] [16] 


$$
\begin{aligned}
E(1) & =\frac{1}{2}\left(D_{1}\right)\left(\phi^{2}\right) m c^{2} \\
& =\left(5 \phi^{2} / 2\right) m c^{2} \\
& =E(D) .
\end{aligned}
$$

On the other hand $D_{2}=\phi^{3}$ is related to the ordinary energy and given by [13] [14] [15] [16]

$$
\begin{aligned}
E(2) & =\frac{1}{2}\left(D_{2}\right)\left(\phi^{2}\right) m c^{2} \\
& =\left(\phi^{5} / 2\right) m c^{2} \\
& =E(O) .
\end{aligned}
$$

Adding $\mathrm{E}(1)$ and $\mathrm{E}(2)$ one finds the most famous ad recognized equation of physics, namely [13] [14] [15] [16]

$$
\begin{aligned}
E & =E_{1}+E_{2} \\
& =\left(5 \phi^{2} / 2\right)\left(m c^{2}\right)+\left(\phi^{5} / 2\right)\left(m c^{2}\right) \\
& =m c^{2} \\
& =E \text { (Einstein) } .
\end{aligned}
$$

Incidentally the preceding analysis entails several deep philosophical conclusions of which we may mention the following:

a) Time as well as gravity are emergent phenomena caused by quantum entanglement of the Hardy type [9] [10].

b) Quantum entanglement is a consequence of the fractal character of spacetime and in particular the zero set and the empty set [9] [10].

\section{Epilogue and Discussion}

Recent triple-slit experiments revealed beyond any reasonable doubt that light may traverse the set up in unexpected looped form. These loops, which are reminiscent of the Peano-Hilbert model of spacetime (compare Figure 1 and Figure 2 of the present paper) are interpreted here as being the signature of the very structure of real quantum spacetime brought to the fore. It is found that a quantum spacetime dimension consistent with this picture and Cantorian quantum gravity is a fractal Kaluza-Klein dimension $D=5+\phi^{3}$. In turn the corresponding total energy density of the universe follows from that to be [13] [14] [15] [16]

$$
\begin{aligned}
E & =\left(5+\phi^{3}\right)\left(\frac{1}{2}\right)(\phi 2) m c^{2} \\
& =m c^{2}
\end{aligned}
$$

where $\phi$ is the Sigalotti topological speed of light [13] [14] [15] [16]. This Einstein maximal energy can thus be considered to consist of two parts. The first part

$$
E(O)=(5)\left(\phi^{2}\right) / 2 m c^{2}
$$

is clearly the dark energy of the cosmos while the second part

$$
E(D)=\left(\phi^{5} / 2\right) m c^{2}
$$


is the measured ordinary energy density which accounts to only 4.5 percent of the total energy [9] [10].

It is appropriate in view of the above to conclude this conclusion with some remarkable insights of a scientist with a considerable intuitive mind that was always ahead of his mathematics. I mean of course one of my teachers and mentors who is alas no longer with us, the late Nobel Laureate Ilya Prigogine who wrote round 1987 [11]:

"Probably the most fascinating aspect involved in the transition from dynamics to thermodynamics is the deep change in the structure of spacetime".

It seems that Prigogine's insight also applies to the transition from classical to quantum spacetime [1]-[17].

\section{Conclusions}

Let us recapitulate what we consider are the most important insights and conclusions made explicitly and implicitly in the present paper:

a) While a one-slit experiment with light reveals its classical deadly straight path, the two-slit experiment reveals clearly its wave character giving rise to a well known historical controversy. It is really the three-slit experiment which in our opinion, settles the said controversy and shows that light and spacetime geometry and topology are inseparable on a fundamental level [17] [18] [19] [20] [21].

b) There is a very instructive analogy between the preceding point and chaos theory. We know that there is no one point chaos game while the two points chaos game leads to a single attracting point. It is only the three and four points chaos game [19] [20] [21] that leads to interference like pattern and is used to illustrate the two-slit experiment with quantum particles. For this reason we can be reasonable sure that the superficially paradoxical outcome of the two-slit experiment is put aside by accepting a Peano-Hilbert micro spacetime or accepting that the result of the three-slit experiment is generic.

c) Finally we stress that the results of the three-slit experiment should by no means be that surprising if we consider that while in the classical world light moves in straight lines, in Einstein's curved Riemannian world the path of the light is also curved in the large structure of the universe. It follows then that light will have a zigzag Zitterbewegung-like path on the quantum "micro" scale for which a Peano-Hilbert spacetime is merely a coarse grain of Cantorian-fractal spacetime as demonstrated by Ji-Huan He several years ago [18].

For all the above mentioned points we think the result in Refs. [1] [2] [3] is an experimental confirmation of the theory of Cantorian-fractal spacetime [9]-[21] and consequently a confirmation of extra spacetime dimensions as well as dark energy [15]-[21].

\section{References}

[1] Magana-Loaiza, O.S., De Leon, I., Mirhosseini, M., et al. (2016) Exotic Looped Trajectories in the Three-Slit Interference. Arxiv:1610.0858V1[quant-Ph]. 
[2] Sawant, R., Samuel, J., Sinha, A., Sinha, S. and Sinha, U. (2014) Nonclassical Path in Quantum Interference Experiments. Physical Review Letters, 113, 120406. https://doi.org/10.1103/PhysRevLett.113.120406

[3] Zyga, L. (2017) Physicists Detect Exotic Looped Trajectories of Light in Three-Slit Experiment. Physics Org. https://phys.org/news/2017-01-physicists-exotic-looped-trajectories-three-slit.html

[4] El Naschie, M.S. (2004) A Review of E-Infinity Theory and the Mass Spectrum of High Energy Particle Physics. Chaos, Solitons \& Fractals, 19, 209-236. https://doi.org/10.1016/S0960-0779(03)00278-9

[5] El Naschie, M.S. (2005) The Two-Slit Experiement as the Foundation of E-Infinity of High Energy Physics. Chaos, Solitons \& Fractals, 25, 509-514. https://doi.org/10.1016/j.chaos.2005.02.016

[6] El Naschie, M.S. (1993) On Turbulence and Complex Dynamics in a Four Dimensional Peano-Hilbert Space. Journal of the Franklin Institute, 330, 183-198. https://doi.org/10.1016/0016-0032(93)90029-T

[7] Ord, G. (1983) Fractal Space-Time: A Geometric Analogue of Relativistic Quantum Mechanics. Journal of Physics A: Mathematical and General, 16, 1869-1884. https://doi.org/10.1088/0305-4470/16/9/012

[8] El Naschie, M.S. (1994) Young Double-Slit Experiment, Heisenberg Uncertainity Principle and Cantorian Spacetime. Chaos, Solitons \& Fractals, 4, 403-409. https://doi.org/10.1016/0960-0779(94)90053-1

[9] Marek-Crnjac, L. and He, J.-H. (2013) An Invitation to El Naschie's Theory of Cantorian Spacetime and Dark Energy. International Journal of Astronomy and Astrophysics, 3, 464-471. https://doi.org/10.4236/ijaa.2013.34053

[10] Helal, M.A., Marek-Crnjac, L. and He, J.-H. (2013) The Three Page Guide to the Most Important Results of M. S. El Naschie's Research in E-Infinity Quantum Physics and Cosmology. Open Journal of Microphysics, 3, 141-145. https://doi.org/10.4236/ojm.2013.34020

[11] Prigogine and Elskens, Y. (1987) Irreversibility, Stochasticity and Non-Locality in Classical Dynamics. In: Hiley, B. and Peat, D., Eds., Quantum Implications, Routledge, London, 205-223.

[12] El Naschie, M.S. (1998) Some Tentative Proposals for the Experimental Verification of Cantorian Micro Spacetime. Chaos, Solitons \& Fractals, 9, 143-144. https://doi.org/10.1016/S0960-0779(97)00175-6

[13] El Naschie, M.S. (2016) Cantorian-Fractal Kinetic Energy and Potential Energy as the Ordinary and Dark Energy Density Respectively. Natural Science, 8, 511-540. https://doi.org/10.4236/ns.2016.812052

[14] El Naschie, M.S. (2017) Quantum Disentanglement as the Physics behind Dark Energy. Open Journal of Microphysics, 7, 1-27. https://doi.org/10.4236/ojm.2017.71001

[15] El Naschie, M.S. (2016) On a Fractal Version of Witten's M-Theory. Journal of Astronomy \& Astrophysics, 6, 135-144. https://doi.org/10.4236/ijaa.2016.62011

[16] El Naschie, M.S. (2016) The Emergence of Spacetime from the Quantum in Three Steps. Advances in Pure Mathematics, 6, 446-454. https://doi.org/10.4236/apm.2016.66032

[17] El Naschie, M.S. (2017) The Quantum Triple-Slit Experiment and Dark Energy. Open Journal of Microphysics, 7, 31-35. https://doi.org/10.4236/ojm.2017.72002

[18] He, J.-H. (2009) Hilbert Cube Model for Fractal Spacetime. Chaos, Solitons \& Fractals, 42, 2754-2759. https://doi.org/10.1016/j.chaos.2009.03.182 
[19] Iovane, G. and Nada, S.I. (2009) Strange Non-Dissipative and Non-Chaotic Attractors and Palmer's Deterministic Quantum Mechanics. Chaos, Solitons \& Fractals, 42, 641-642. https://doi.org/10.1016/j.chaos.2008.11.024

[20] El Naschie, M.S. (1994) Iterated Function System, Information and the Two-Slit Experiment of Quantum Mechanics. Chaos, Solitons \& Fractals, 4, 1965-1968. https://doi.org/10.1016/0960-0779(94)90011-6

[21] El Naschie, M.S. (1994) Quantum Measurement, Diffusion and Cantorian Geodesics. Chaos, Solitons \& Fractals, 4, 1235-1247.

https://doi.org/10.1016/0960-0779(94)90034-5

Submit or recommend next manuscript to SCIRP and we will provide best service for you:

Accepting pre-submission inquiries through Email, Facebook, LinkedIn, Twitter, etc. A wide selection of journals (inclusive of 9 subjects, more than 200 journals) Providing 24-hour high-quality service User-friendly online submission system Fair and swift peer-review system Efficient typesetting and proofreading procedure Display of the result of downloads and visits, as well as the number of cited articles Maximum dissemination of your research work

Submit your manuscript at: http://papersubmission.scirp.org/

Or contact opj@scirp.org 